\title{
Optimization and Performance of the ATLAS Tau Trigger with Cosmics Data
}

\author{
Mansoora Shamim \\ University of Oregon \\ On behalf of the ATLAS Collaboration \\ Oct 6, 2009
}

I I th ICATPP Conference

Como, Italy

5-9 October 2009
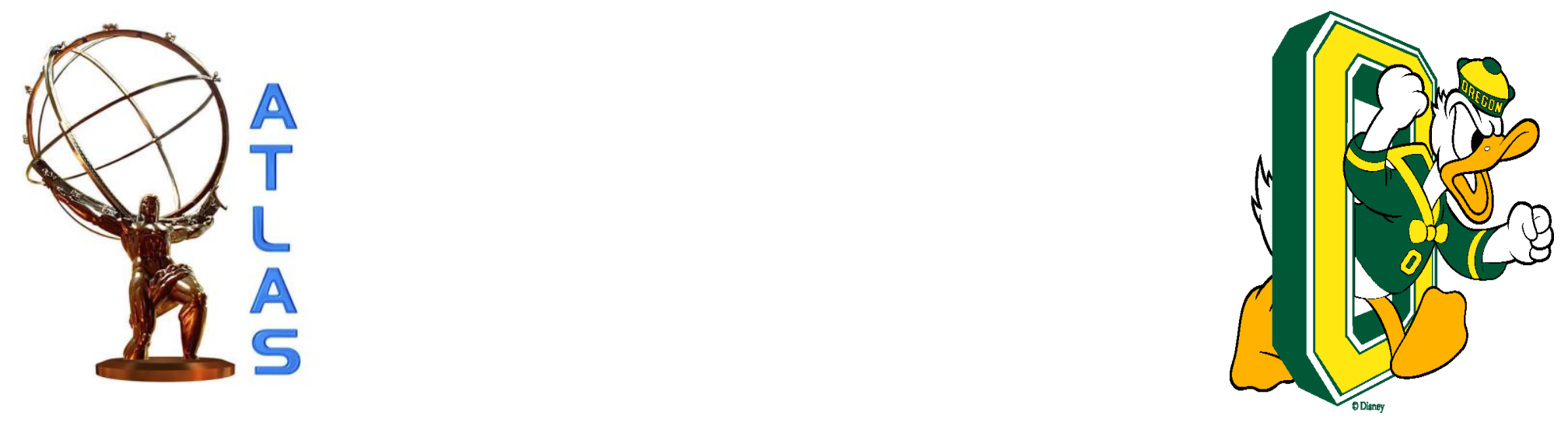


\section{Outline}

- Motivation

- ATLAS detector and trigger system

- Tau trigger at ATLAS experiment

- Performance and optimization of tau trigger

- Cosmics data taking

- Summary 


\section{Motivation}

- What is so special about tau

- the heaviest of the known leptons $\left(m_{\tau}=1776.84 \pm\right.$ $0.17 \mathrm{MeV}$ )

- large number of decay modes

- local, isolated deposition of energy

- A pure sample of taus will

- provide understanding of the detector using standard model processes $(\mathrm{W} \rightarrow \tau \nu$ and $\mathrm{Z} \rightarrow \tau \tau)$

- help search for Higgs boson and Supersymmetry

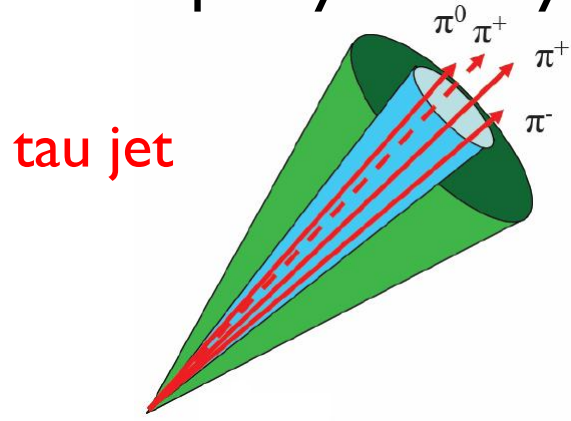

- Decay modes of tau

- leptonic

$$
\begin{aligned}
& \tau^{ \pm} \rightarrow \mathrm{e}^{ \pm}+v+v \\
& \tau^{ \pm} \rightarrow \mu^{ \pm}+v+v
\end{aligned}
$$

- hadronic

- I-prong

$$
\begin{aligned}
& \tau^{ \pm} \rightarrow \pi^{ \pm}+v \\
& \tau^{ \pm} \rightarrow \pi^{ \pm}+v+n \pi^{\circ} \\
& , n \geq I
\end{aligned}
$$

- 3-prong

- $\tau^{ \pm} \rightarrow 3 \pi^{ \pm}+v$

$$
\text { - } \tau^{ \pm} \rightarrow 3 \pi^{ \pm}+v+n \pi^{\circ}
$$

$$
\text { (4.5\%) , } \mathrm{n} \geq 1
$$

- other $\sim 5 \%$

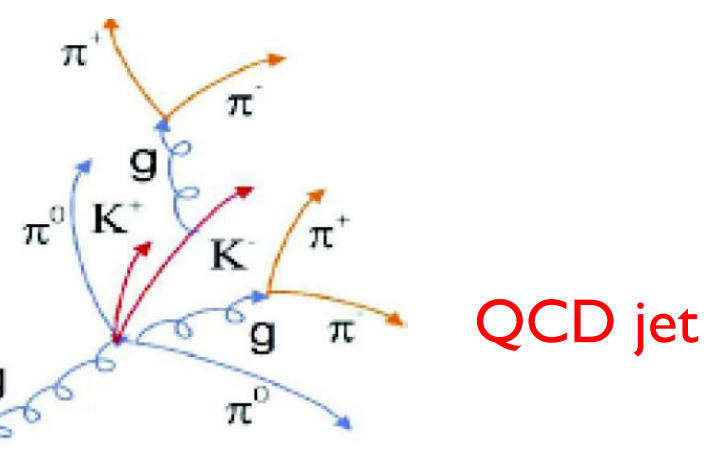




\title{
ATLAS Detector
}

- A general purpose detector

- Trackers

- Pixel

- Silicon microstrip tracker (SCT)

- Transition radiation tracker (TRT)

- Solenoid

- 2T magnetic field

- Calorimeter

- Electromagnetic (EM)-Liquid Argon (LAr)

- Hadronic (HAD)

scintillating tiles in the central barrel, LAr in end caps (EC)

- Muon Spectrometer

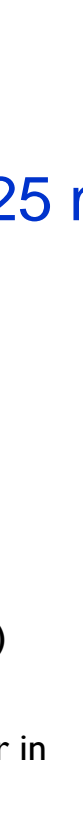

- excellent momentum resolution

- independent momentum measurement at high transverse momentum

- Three large superconducting toroids

- one barrel and two EC

- eight-fold azimuthal symmetry around calorimeter

- I.2T magnetic field

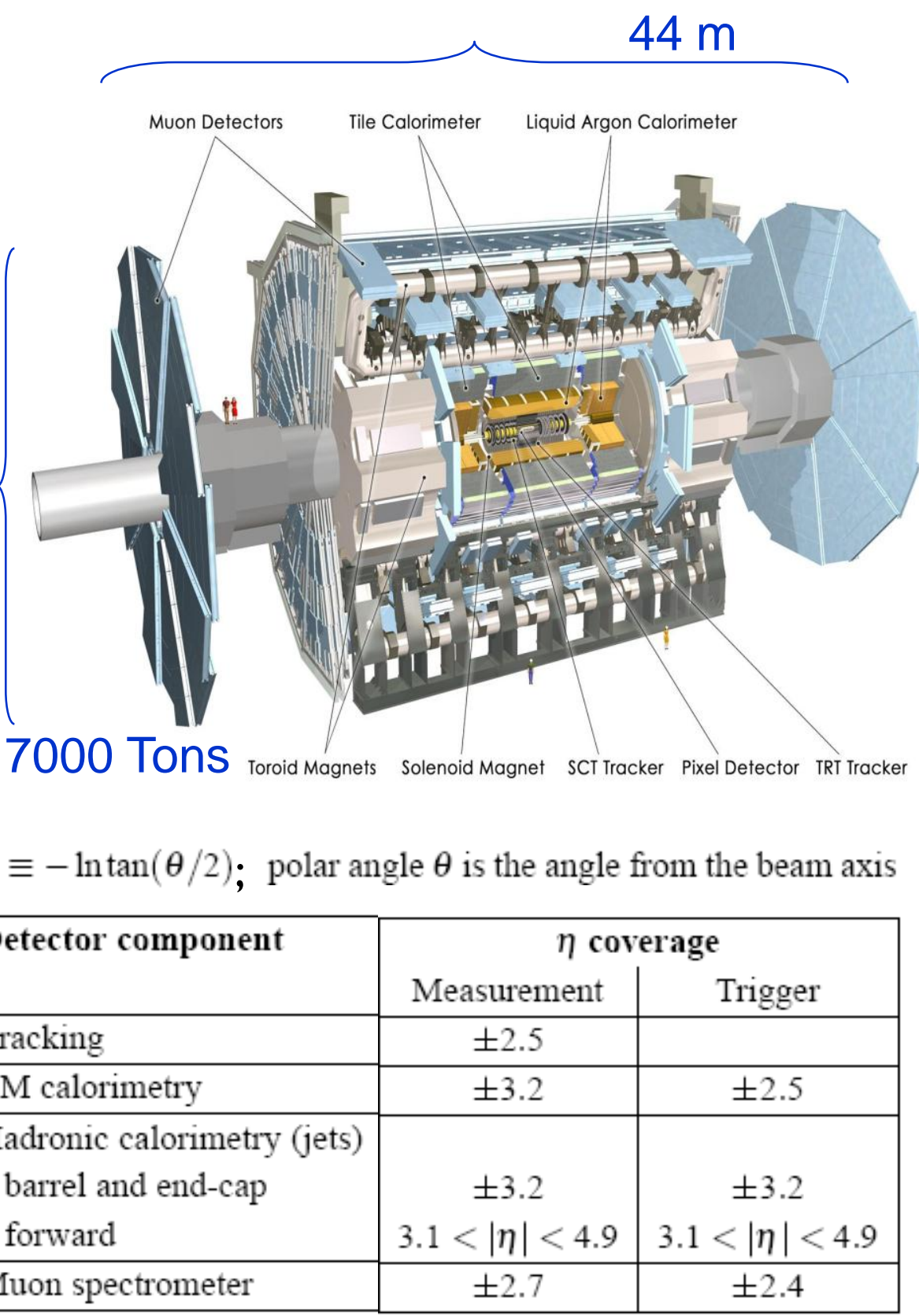

The ATLAS Collaboration, G. Aad et al., The ATLAS Experiment at the CERN Large Hadron Collider, JINST 3 (2008) S08003. 


\section{ATLAS Trigger System}

- Three level trigger system

- Level 1 (L1)

- Hardware-based

- reduced granularity from subset of detectors

- calorimeter and muon

- identifies Regions-of-Interest (Rols)

- decision time $\sim 2.5 \mu \mathrm{s}$

- Level 2 (L2)

- Software-based, seeded by Rols

- full granularity-includes inner detectors

- decision time $\sim 40 \mathrm{~ms}$

- Event Filter (EF)

- uses offline analysis procedure

- fully built events

- processing time $\sim 4 s$

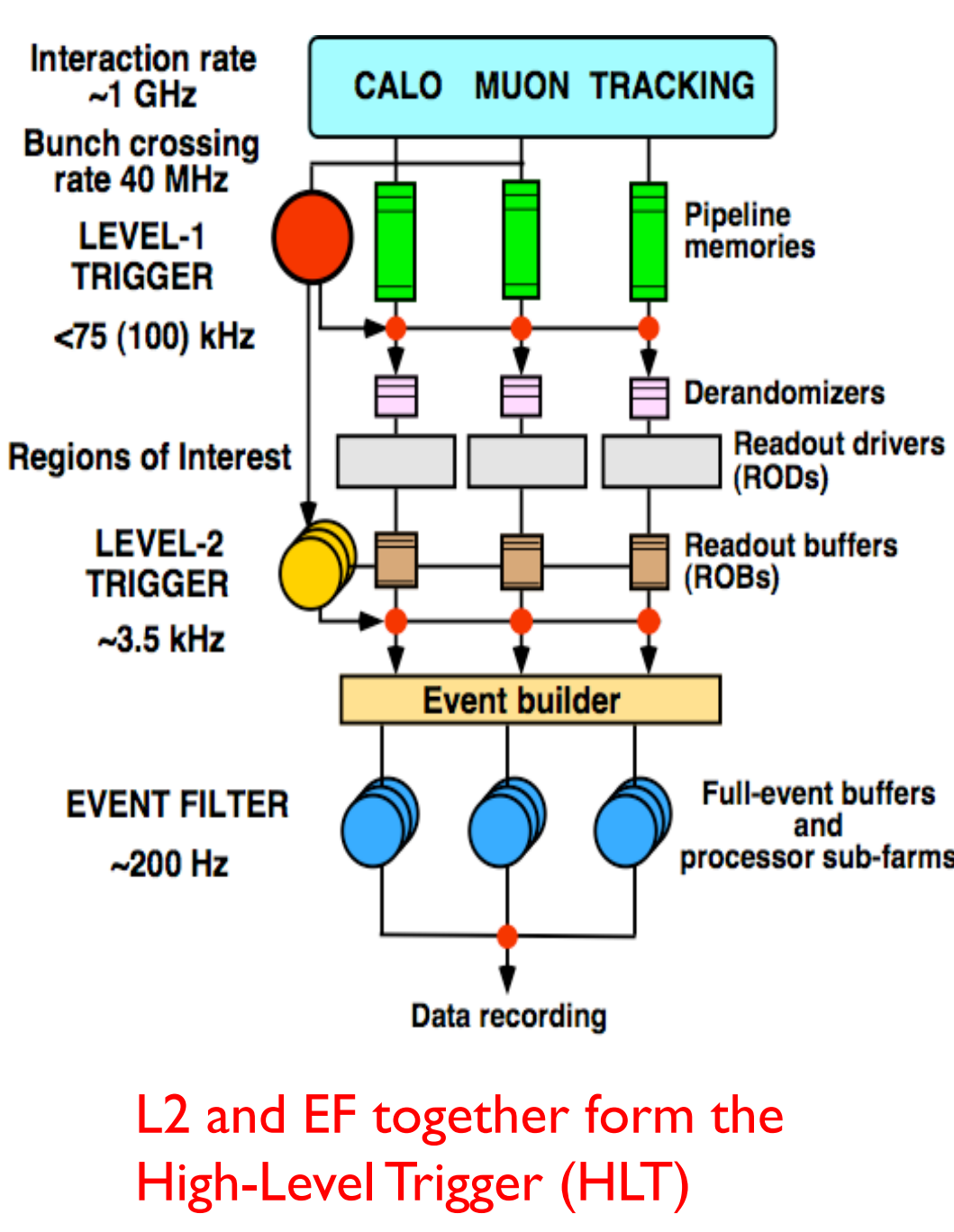




\section{Tau High Level Trigger}

- Level 2 (L2)

- seeded by Rol at Level 1

- information from both calorimeter and tracking is used
- Event Filter

- seeded by Rol from L2

- algorithms similar to offline tau reconstruction are used to find good tau candidates

- L2 and EF make use of track multiplicity, narrowness and isolation criteria to increase the purity

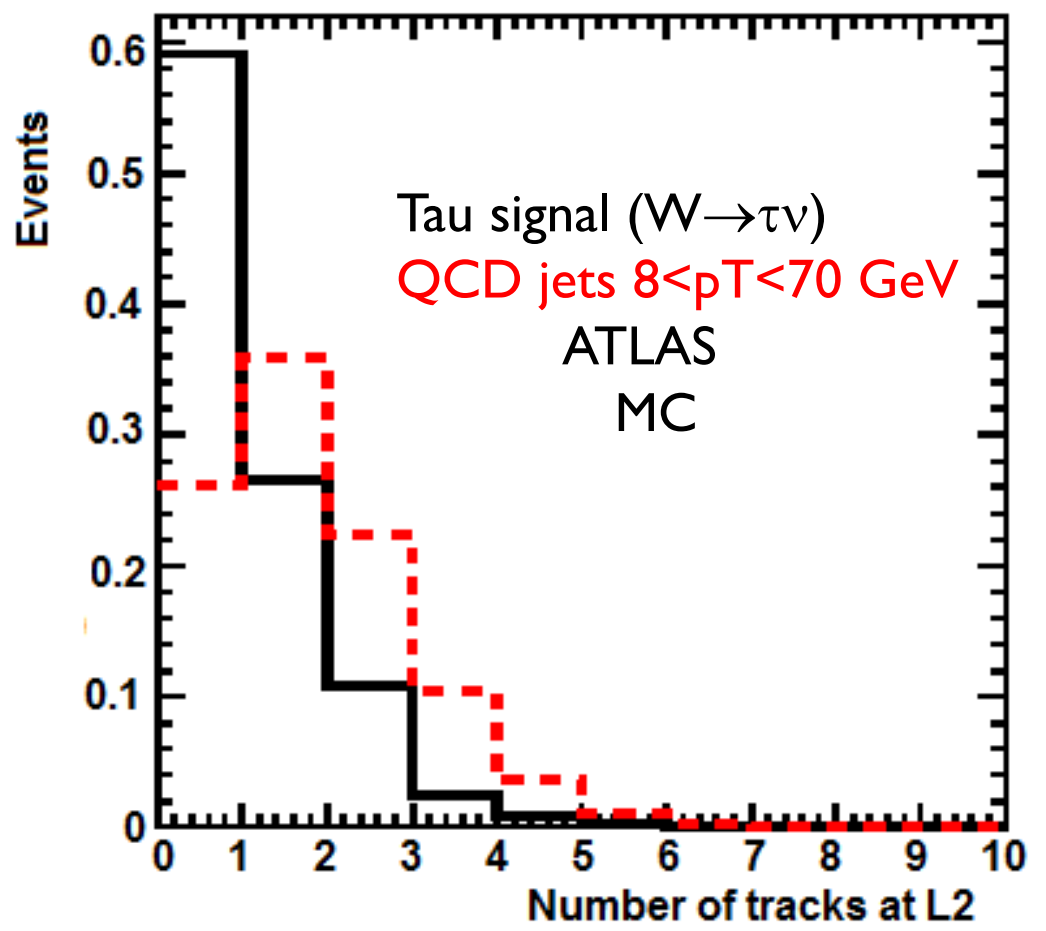

CERN-OPEN-2008-020

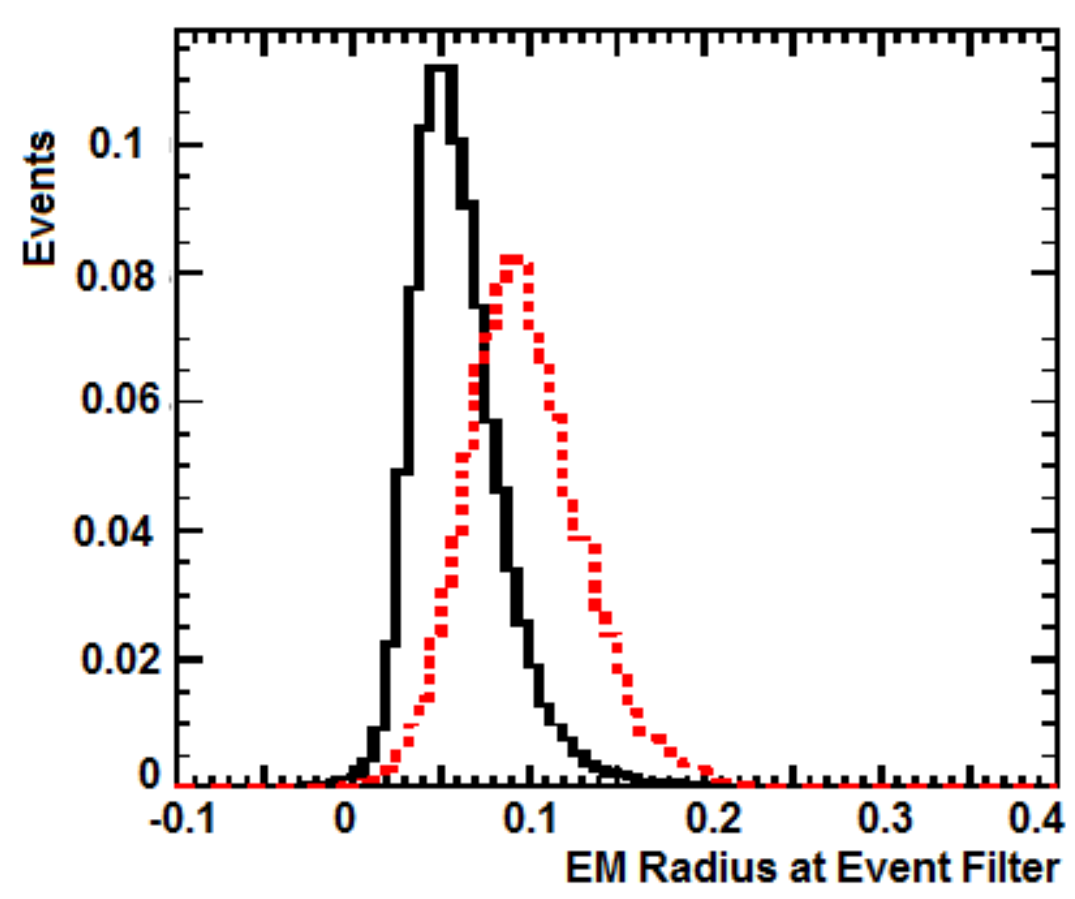




\section{Tau Trigger Menu}

- Menu

- A table which specifies the threshold and selection criteria at each trigger level

- Separate menus designed to deal with different luminosities

- motivated by physics requirements

- control background rates while keeping the signal efficiency high

- At $10^{31} \mathrm{~cm}^{-2} \mathrm{~s}^{-1}$

- tau trigger will be used in combination with missing transverse energy (MET), electron and muon triggers

- enriched sample of $W \rightarrow \tau v$

- understanding of detector and tau reconstruction and identification software
- Tau Trigger menu for $10^{31} \mathrm{~cm}^{-2} \mathrm{~s}^{-1}$ at $10 \mathrm{TeV}$

- errors are statistical only- rates calculated using multi-jet samples

- ATL-DAQ-CONF-2008-002

\begin{tabular}{|l|l|l|c|}
\hline \hline Menu & Goal & Trigger & Unprescaled Rate $[\mathrm{Hz}]$ \\
\hline \hline Single Tau & Searches at high $P_{T}$ & tau50_loose & $0.9 \pm 0.5$ \\
tau+lepton & $Z \rightarrow \tau \tau$ & tau12_loose_e10_loose & $2.0 \pm 0.7$ \\
tau+MET & $W \rightarrow \tau \nu, t \bar{t}, Z \rightarrow \tau \tau$ & tau16_loose+xe25 & $6.9 \pm 1.2$ \\
tau+jets & $t \bar{t}$ & tau16i_loose+3j40 & $2.2 \pm 0.7$ \\
\hline \hline
\end{tabular}

- At $\sim 10^{33} \mathrm{~cm}^{-2} \mathrm{~s}^{-1}$

- tight identification requirements, increased $E_{T}$ threshold or tau trigger combined with lepton, jet, MET triggers

- sample used to search for new physics

- $\mathrm{H}^{ \pm} \rightarrow \tau \nu, \mathrm{H} \rightarrow \tau \tau, Z^{\prime}$, decay chains of SUSY particles 


\section{Tau Trigger Performance}

- Efficiency measured using $Z \rightarrow \tau \tau$ sample

$$
\text { Efficiency }=\frac{\# \text { of truth matched reconstructed taus that fired a trigger }}{\# \text { of truth matched reconstructed taus }}
$$

ATL-DAQ-PROC-2009-027

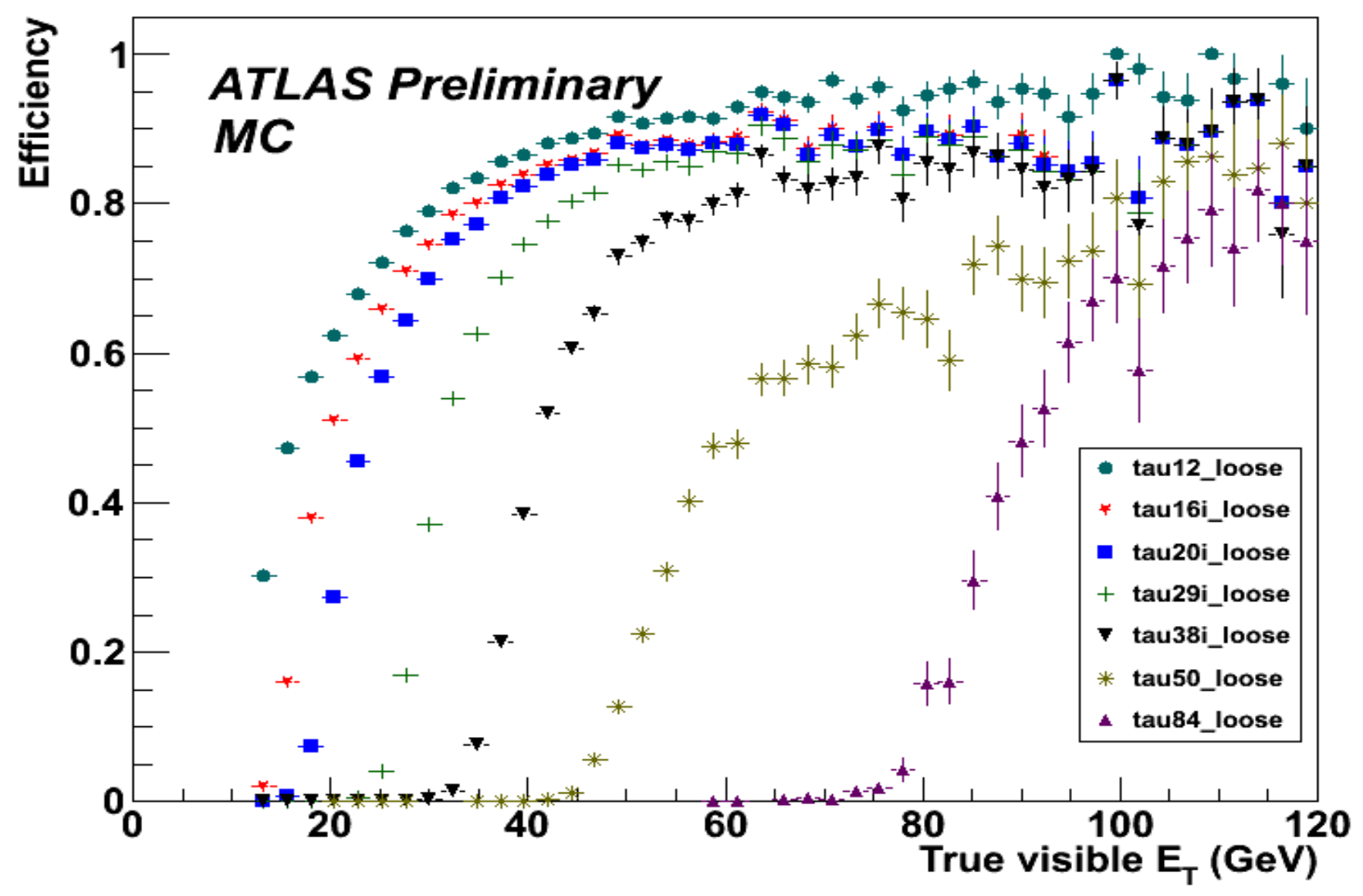




\section{Plans to Measure Tau Trigger Efficiency in}

\section{Early Data}

- Single tau trigger efficiency can be measured using $\mathrm{Z} \rightarrow \tau(\mathrm{e} / \mu) \tau(\mathrm{had})$

- tag and probe method

- require tag electron to pass e/ $\mu$ trigger

- what fraction of probes pass single tau trigger

direct measurement but low statistics

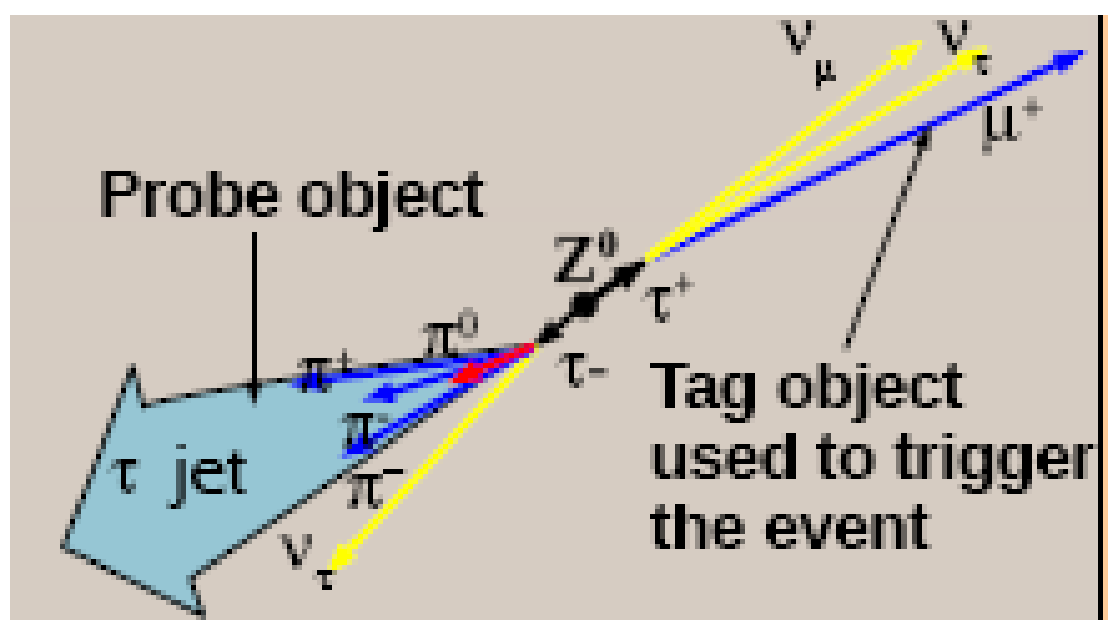

- QCD events (fake taus)

- higher statistics

- tight offline selections are applied to select good taus

- Top events

- events triggered by 3jet trigger

- one of the $W$ decays into tal

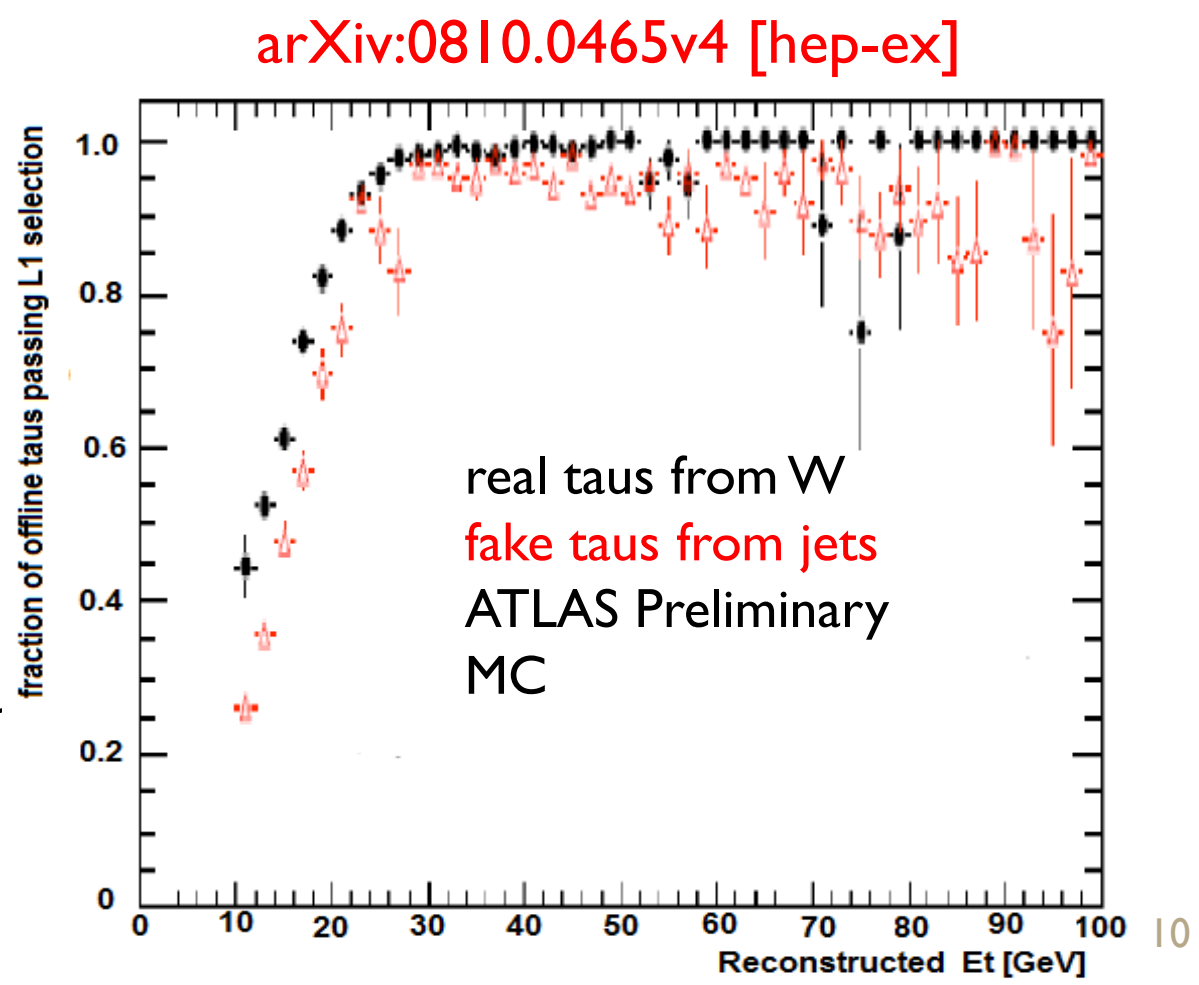
how often this tau fires a tau trigger 


\section{Cosmics-ray Data at ATLAS}




\section{Cosmics Data Taking}

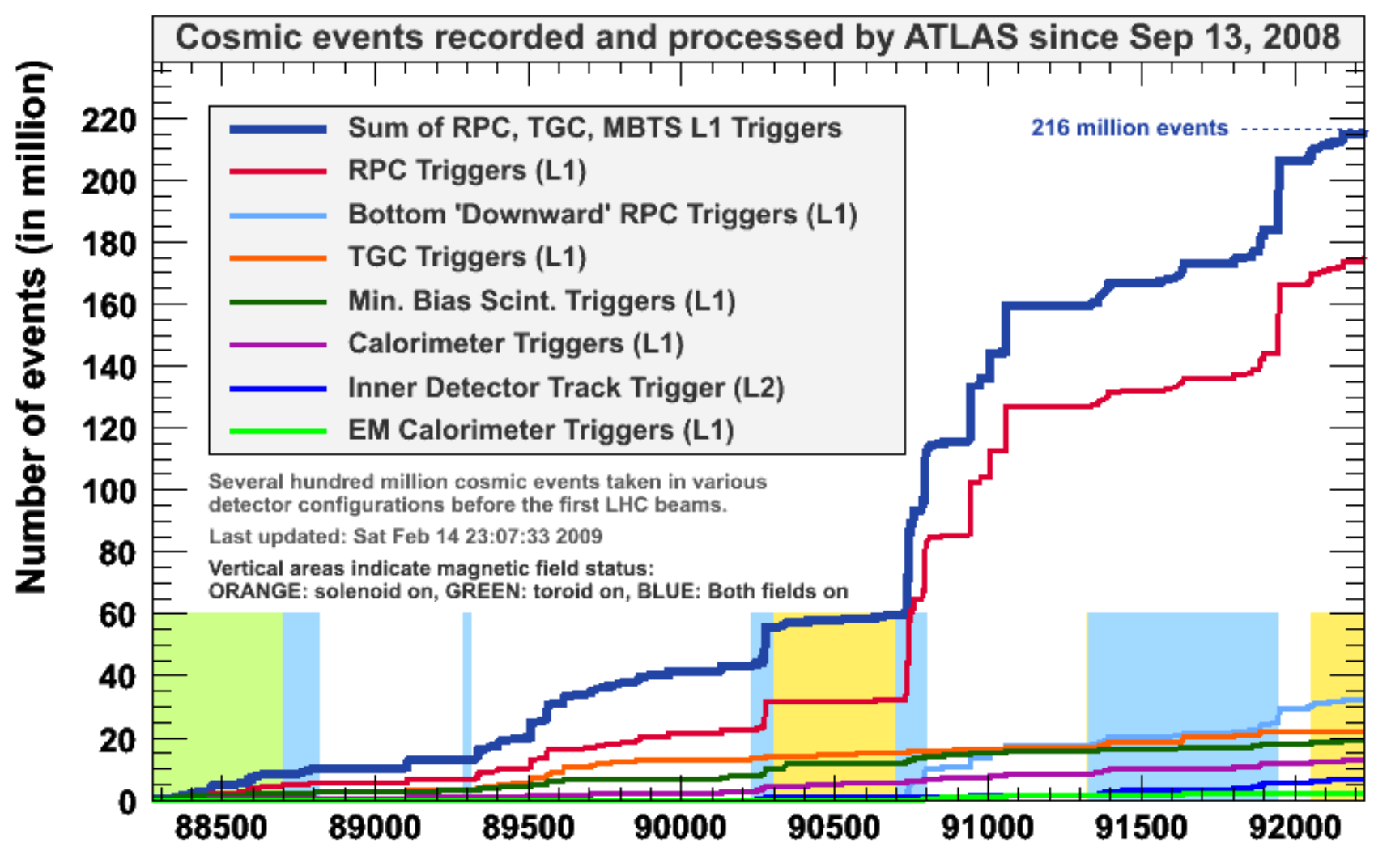

Run number

- ATLAS was ready for LHC data taking in Aug 2008

- Since then global cosmic runs

- $500 \mathrm{M}$ events collected:Aug-Oct 2008 alignment studies

- Single beam events in Sep 2008

- Detector maintenance and repair work: Oct 2008 - Jun 2009

- Global cosmic runs in June 2009

- $100 \mathrm{M}$ events collected

- Combined global cosmic runs to start again in Oct 2009

- used for timing studies 


\section{ATLAS beam splash event (Sep 10,2008)}

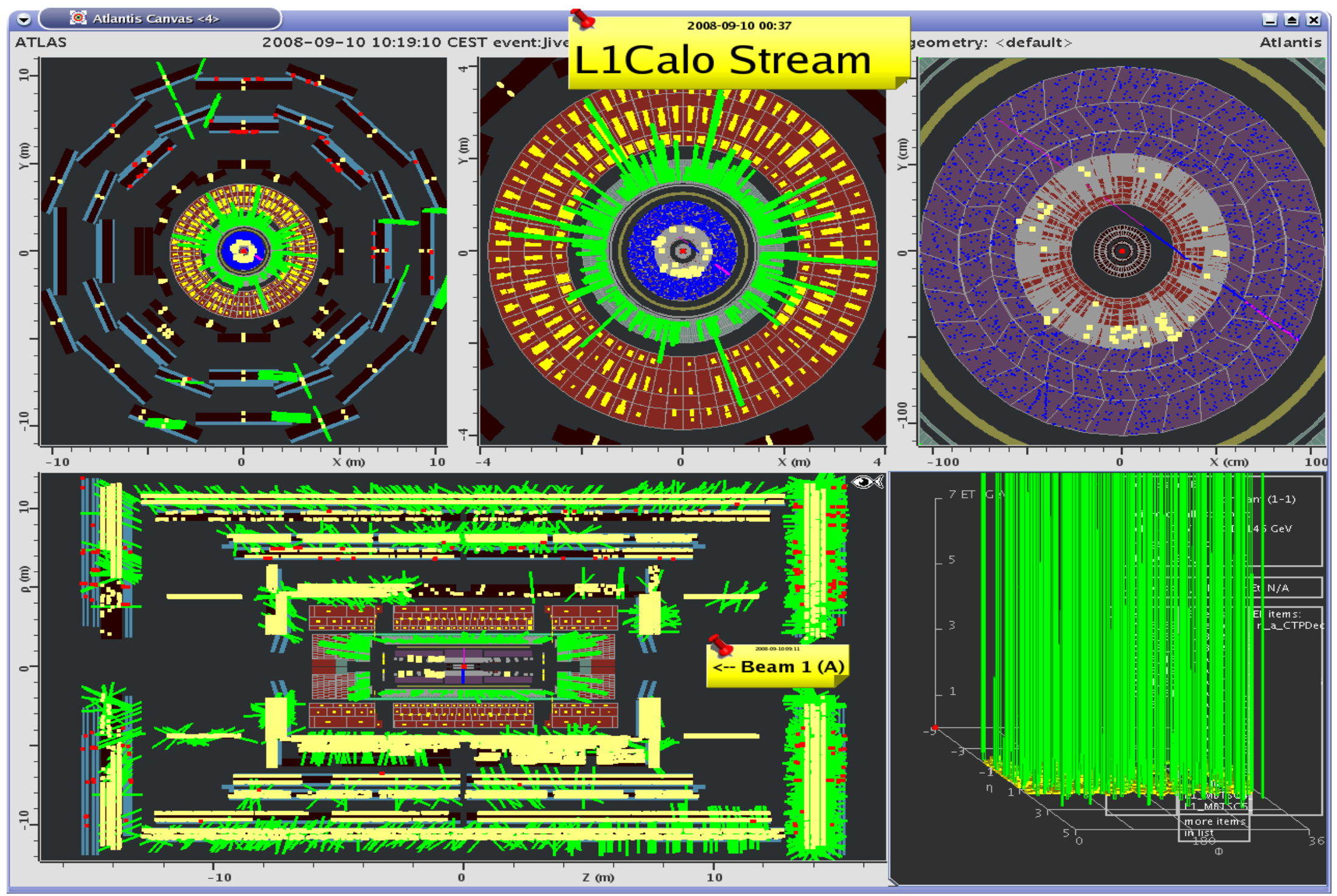

Triggered by LITAU, LI Min. Bias and LIJET 


\section{Cosmic-ray Event Display}

- energy deposition in the calorimeter

- a track reconstructed by tau trigger algorithms

- helpful in commissioning of tau trigger

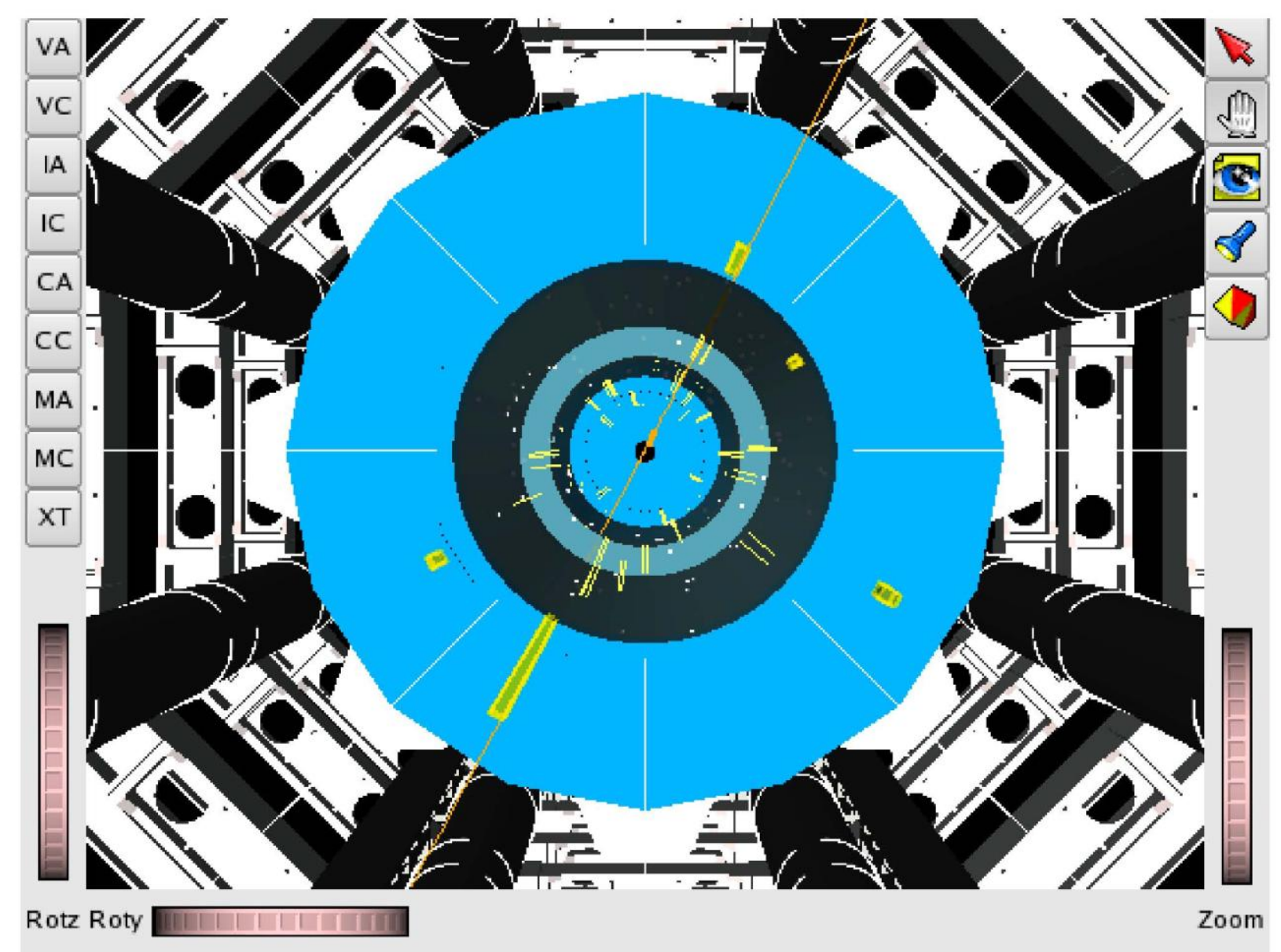




\section{Cosmics Data and Tau Trigger}




\section{Energy Correlation at Level 1(L1)}

- LI calorimeter energy correlation plot showing the energy measured in

- trigger towers used to apply threshold

- calorimeters cells

- Different electronics and subject to different calibrations

- Sharp cut-off due to trigger threshold of $5 \mathrm{GeV}$

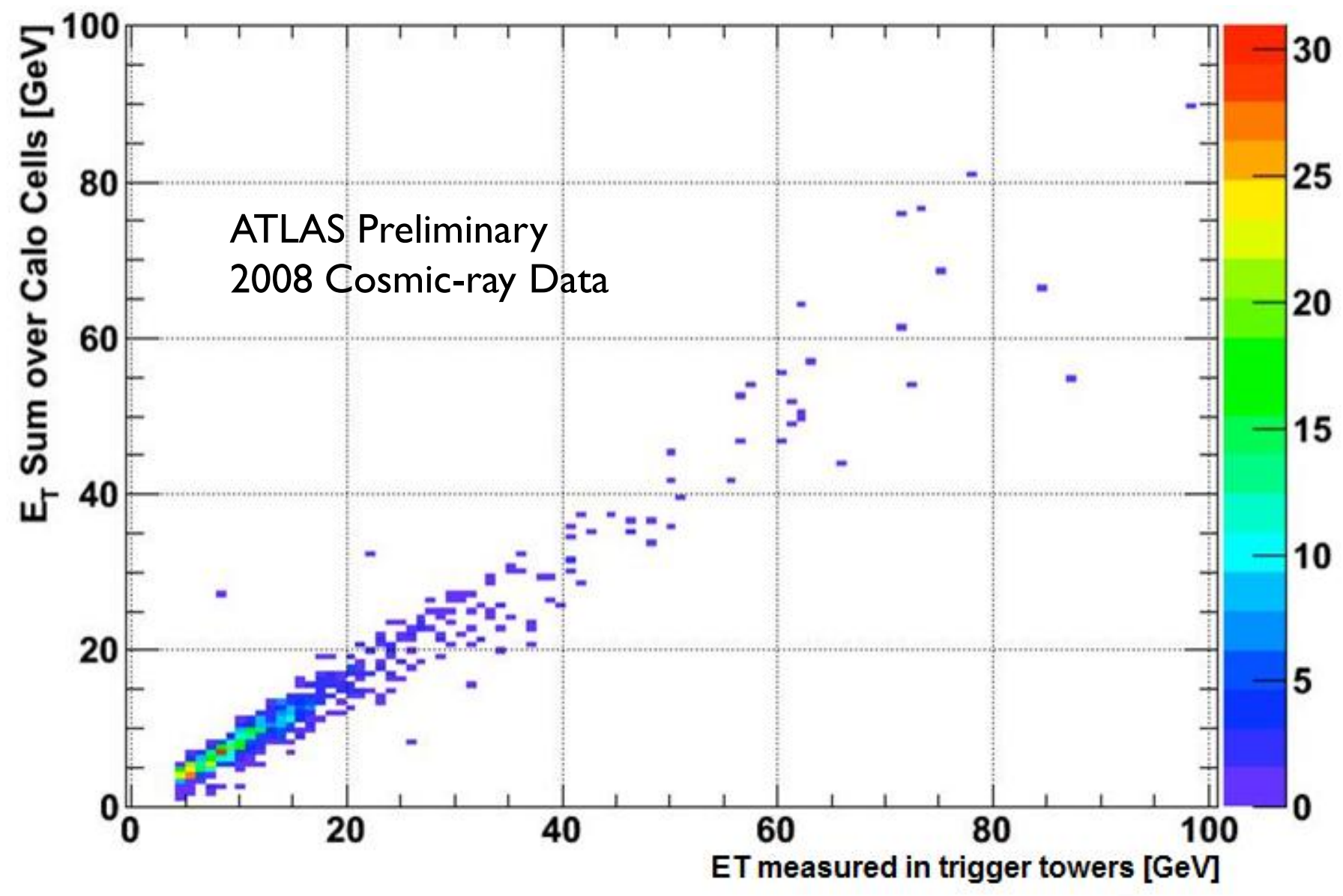




\section{Tau Trigger Data Quality (DQ) Monitoring}

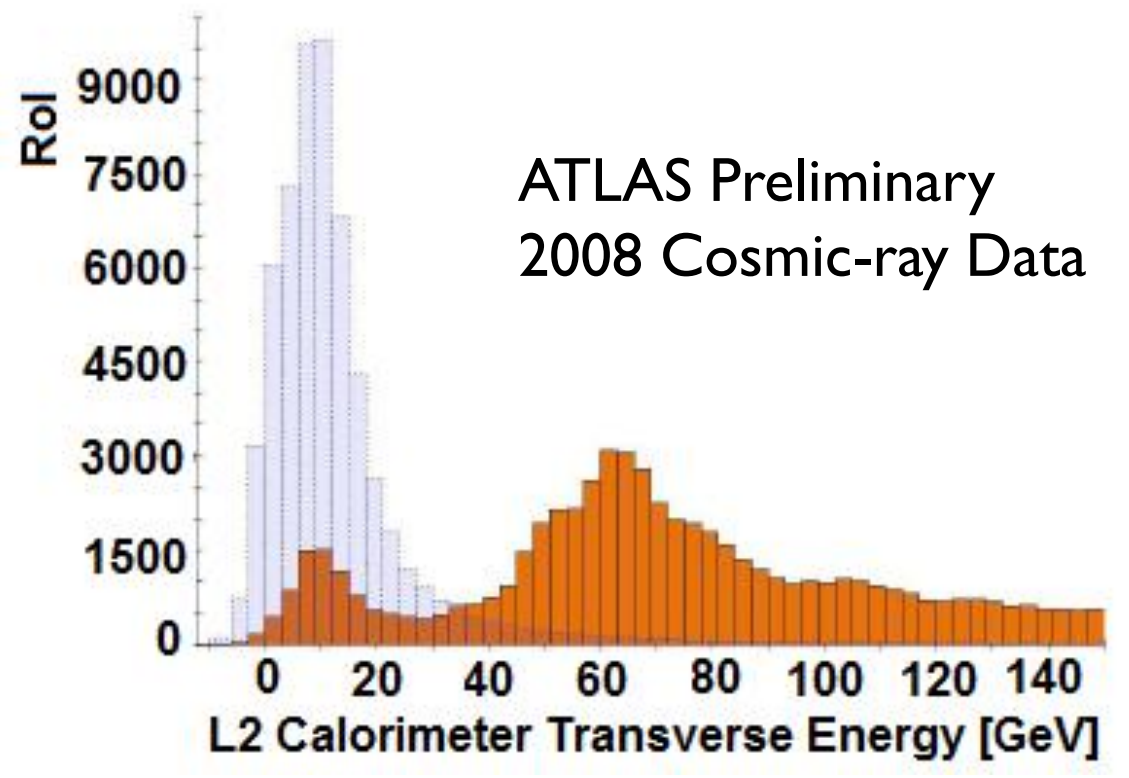

- Online DQ plots

- grey: reference

- green \&\& red: data being taken online

- issues with the total energy deposition

- EM energy distribution looks fine

- problem in HAD energy deposition

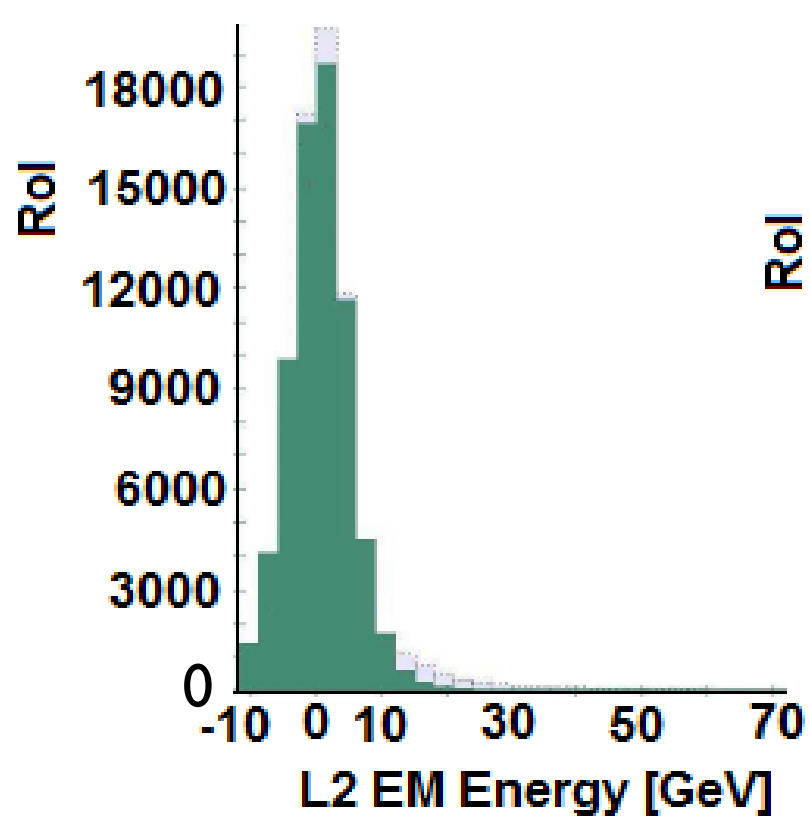

solution: trigger configured properly to cure the problem

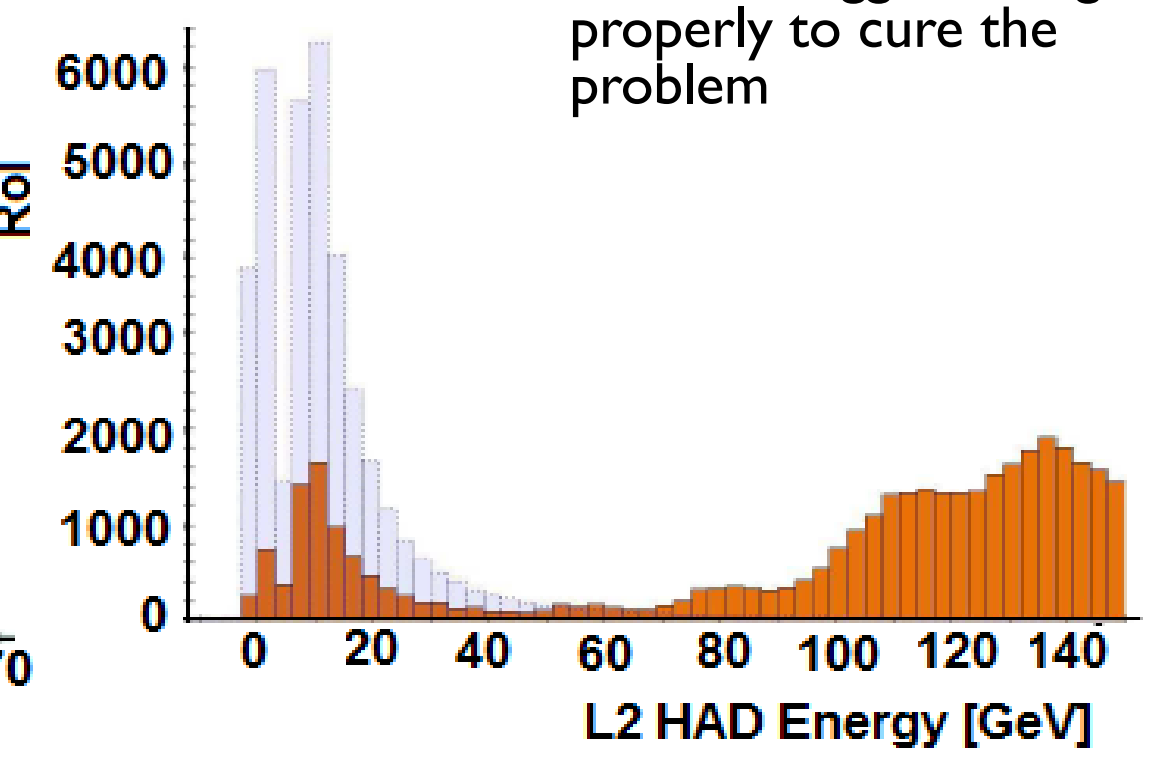




\section{Summary}

- A dedicated tau trigger at ATLAS

- designed and its performance evaluated using simulated events

- commissioned during 2008 cosmics data taking

- has provided valuable tool to understand the detector

- MIP studies

- DQ monitoring

- Ready to collect data with colliding beams

- further understanding of SM processes

- unveil the mysteries through searches for new physics using taus

- effect of improvements implemented in algorithms - based on the studies of 2008 cosmics data 


\section{General Information}

- Talk will be on Tuesday Oct 6, 2009

- Parallel session IX on high energy physics II 14:30-16:00

- Does not explicitly say how long the talk should be

- 90 minutes and 4 talks scheduled

- $20+2$ minutes

- Slide 17 is tentative-plot needs to be approved

- Another ATLAS talk in the same session on trigger monitoring 


\section{MIP Cluster at Event Filter}

- Typical MIP energy deposit

- $200 \mathrm{MeV}$ in LAr (EM)

- $4 \mathrm{GeV}$ in Tile (HAD)

- Uncorrelated behavior seen in EM and HAD energy deposition at $\mathrm{LI}$ in cosmic events

- Helpful to extract properties of MIP

- Large EM energy depositionby energetic photon via Bremsstrahlung

- small HAD energy depositionmuon as MIP

- Large HAD energy deposition

- MIP cluster firing the trigger above $5 \mathrm{GeV}$ threshold

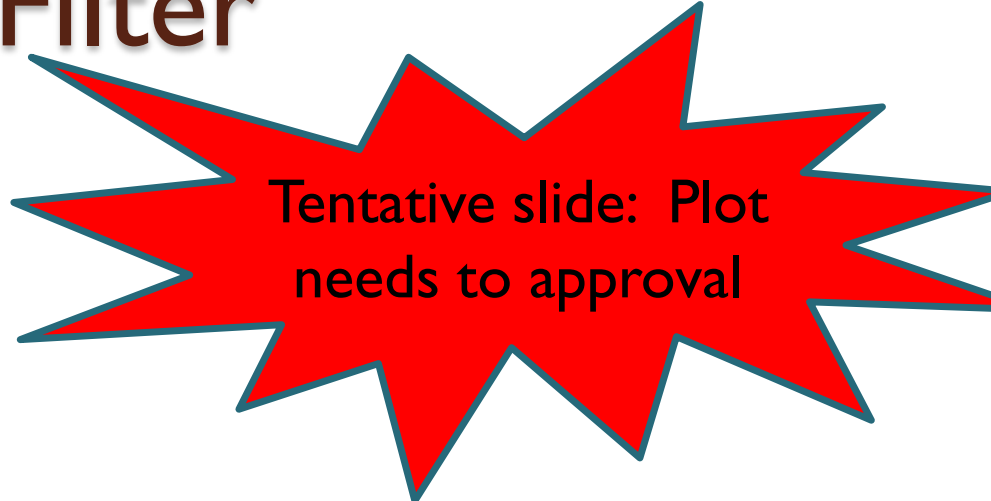

L1 EM seeded (L1 Em $E_{T} \geq 5 \mathrm{GeV}$ and L1 Had $\left.E_{T}=0 \mathrm{GeV}\right)$,

L1 HAD seeded (L1 Em $E_{T}=0 \mathrm{GeV}$ and L1 Had $E_{T} \geq 5 \mathrm{GeV}$ ),

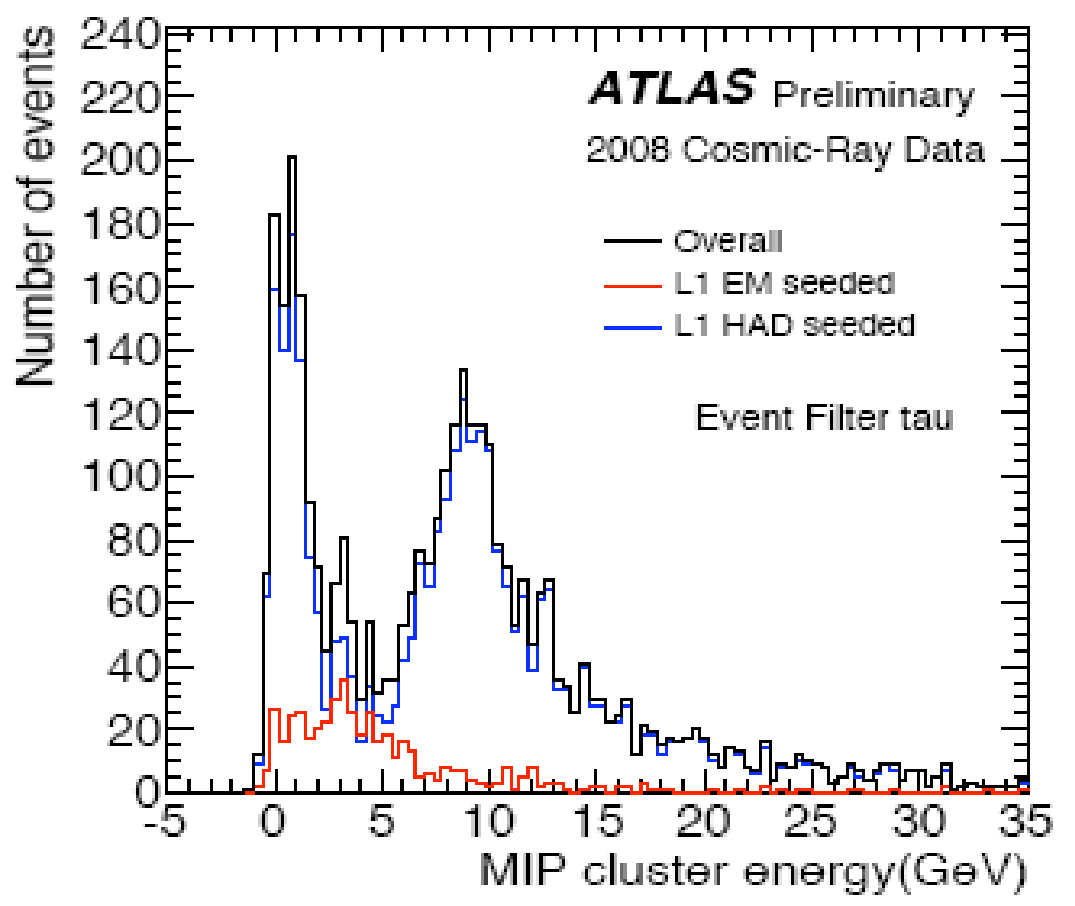

